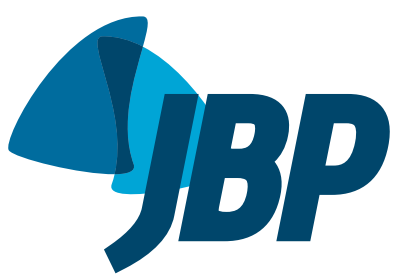

\section{Calcified intracavitary mass: a rare presentation of aspergilloma}

\author{
Edson Marchiori ${ }^{1, a}$, Bruno Hochhegger ${ }^{2, b}$, Gláucia Zanetti1,
}

A 69-year-old woman presented with a 2-year history of recurrent episodes of hemoptysis, one of which was severe, leading to admission to an intensive care unit. She had a history of pulmonary tuberculosis treated 20 years previously. A chest X-ray (Figures $1 \mathrm{~A}$ and $1 \mathrm{~B}$ ) and chest CT (Figures 1C and 1D) showed a thin-walled cavity, containing an ovoid calcified mass, in the upper right lobe. The patient underwent right upper lobectomy. Microscopic examination showed that the mass was a calcified capsule filled with abundant necrotic material, fungal hyphae, and birefringent calcium oxalate crystals. Cultures grew Aspergillus niger. The final diagnosis was pulmonary aspergilloma caused by $A$. niger and presenting as a calcified mass.
A fungus ball or aspergilloma is the most common cause of intracavitary nodules, generally resulting from fungal colonization of pre-existing lung cavities. ${ }^{(1)}$ One feature of $A$. niger infection that is key for the diagnosis is the presence of calcium oxalate crystals, detected by pathological examination. ${ }^{(2,3)}$ Some early reports of aspergillomas mentioned calcification, as identified on chest X-rays, which is related to the presence of calcium oxalate crystals. However, to our knowledge, there have been no reports of aspergilloma presenting as a calcified intracavitary mass identified on computed tomography scans.

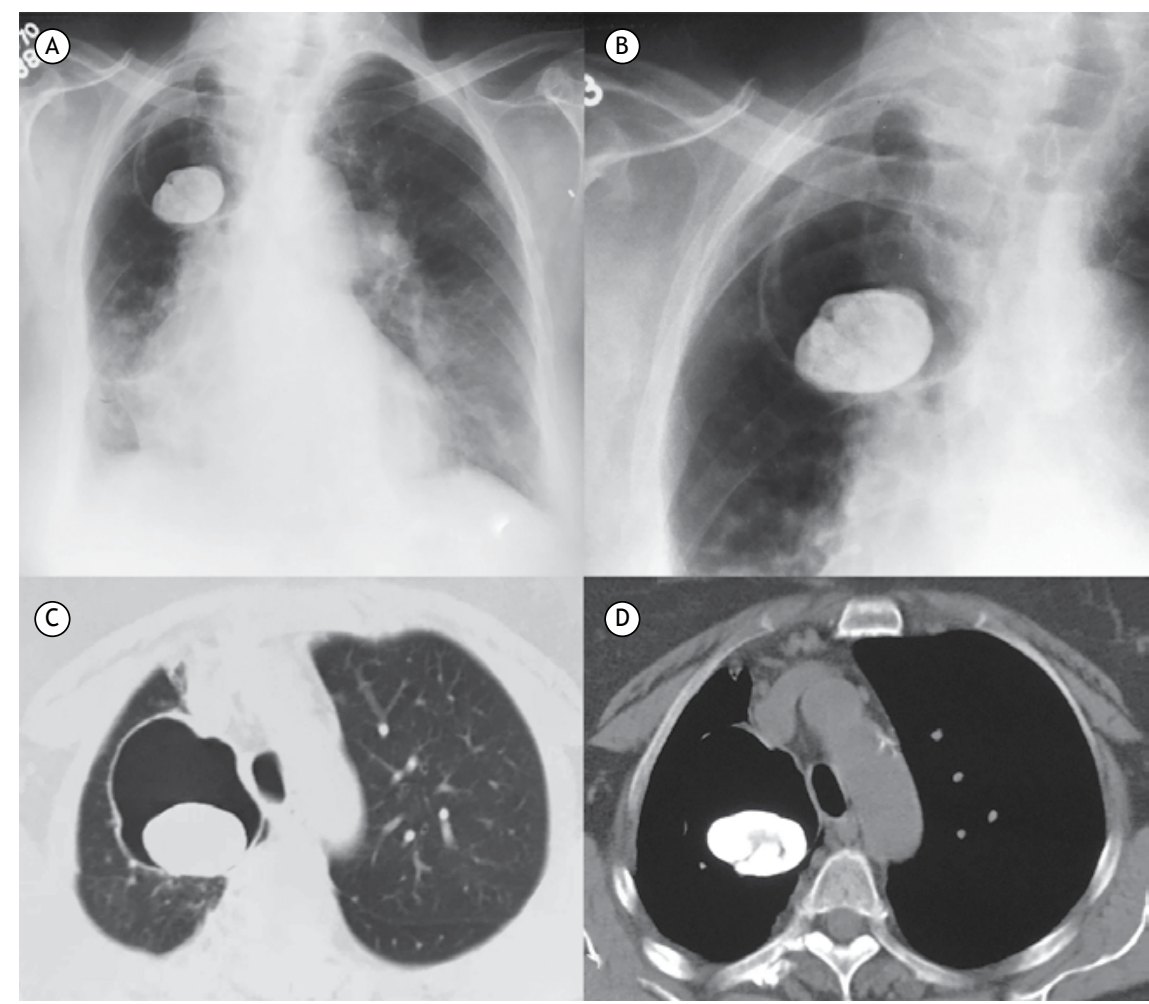

Figure 1. Chest X-ray $(A)$, with a detailed view of the right upper lung region (B), showing a thin-walled cavity in the right upper lobe containing an ovoid calcified mass with a maximum diameter of about $4 \mathrm{~cm}$. Chest CT with lung and mediastinal window settings ( $C$ and $D$, respectively), confirming the presence of the mass inside the cavity.

\title{
REFERENCES
}

1. Marchiori E, Hochhegger B, Zanetti G. Intracavitary nodule. J Bras Pneumol 2016(5);42:309. https://doi.org/10.1590/S180637562016000000223

2. Person AK, Chudgar SM, Norton BL, Tong BC, Stout JE. Aspergillus niger: an unusual cause of invasive pulmonary aspergillosis. J Med
Microbiol. 2010;59(Pt 7):834-8. https://doi.org/10.1099/jmm.0.018309-0

3. Oda M, Saraya T, Wakayama M, Shibuya K, Ogawa Y, Inui T, et al. Calcium oxalate crystal deposition in a patient with Aspergilloma due to Aspergillus niger. J Thorac Dis. 2013;5(4):E174-8. https://doi. org/10.3978/j.issn.2072-1439.2013.08.4

1. Universidade Federal do Rio de Janeiro, Rio de Janeiro (RJ) Brasil. 